\title{
MARTIN'S AXIOM IMPLIES THAT DE CAUX'S SPACE IS COUNTABLY METACOMPACT
}

\author{
WILLIAM G. FLEISSNER ${ }^{1}$
}

\begin{abstract}
De Caux defined a space $S(\mathcal{E})$ and, assuming \&, showed that $S(\mathfrak{L})$ is normal but not countably metacompact. We assume $M A_{\omega_{1}}$ and show that $S(E)$ is countably metacompact.
\end{abstract}

In [dC] Peter de Caux constructed a Dowker space assuming \&. (For a definition of $\&$ and review of the Dowker space problem, see [R].) Peter Nyikos [N] asked whether it was consistent with $\mathrm{MA}+\neg \mathrm{CH}$ that de Caux's space be a Dowker space. Here we answer negatively by proving the title. (Martin's Axiom in the title means $\mathrm{MA}_{\omega_{1}}$ )

Definition. A ladder system, $\mathcal{L}$, is a sequence $\left(L_{\lambda}: \lambda \in \omega_{1} \cap\right.$ LIM) such that each $L_{\lambda}$ is a cofinal subset of $\lambda$ with order type $\omega$.

Definition. Given a ladder system $\mathcal{L}$, we define a space $S(\mathcal{L})$ as follows. The point set of $S(\mathfrak{L})$ is $\omega \times \omega_{1}$. We define a topology on $\omega \times \omega_{1}$ by defining a weak base $\mathscr{B}_{x}$ at each point $x$. A set $U$ will be open iff $x \in U$ implies there is $B \in \mathscr{B}_{x}$, $B \subset U$. If $x$ is of the form $(0, \alpha)$ or $(n, \alpha+1)$, the only element of $\mathscr{B}_{x}$ is $\{x\}$. If $x$ is of the form $(n+1, \lambda)$, where $\lambda \in \operatorname{LIM}$, then $\mathscr{B}_{x}$ is the countable family of sets of the form $\{(n+1, \lambda)\} \cup\left\{\{n\} \times\left(S_{\lambda}-e\right)\right\}$, where $e$ is a finite set.

De Caux assumed \& to get the existence of a ladder system $\mathcal{L}$ with special properties, and used the special properties to show that $S(\mathfrak{L})$ is a Dowker space. Nyikos calls any space of the form $S(\mathcal{L})$ a de Caux's Litmus strip space. Clearly, one can construct $S(\mathcal{L})$ without assuming \&. Further, the properties of $S(\mathfrak{L})$ could vary with $\mathcal{L}$. It is not hard to define $\mathcal{E}$ so that $S(\mathcal{E})$ is a $\sigma$-discrete Moore space, for example. What we are asserting in this paper is that assuming $\mathrm{MA}+\neg \mathrm{CH}$, all spaces of the form $S(\mathcal{E})$ are countably metacompact.

Definition. A space is countably metacompact iff whenever $\left(H_{n}\right)_{n \in \omega}$ is a decreasing sequence of closed sets with empty intersection then there is a sequence $\left(U_{n}\right)_{n \in \omega}$ of open sets with empty intersection satisfying $U_{n} \supset H_{n}$.

Definition. A poset $P$ has property $K$ iff whenever $W$ is an uncountable subset of $P$ there is an uncountable subset $W^{\prime}$ of $W$ of compatible elements. The product of property $K$ posets has property $K$, a fortiori, ccc.

Definition. For $A$ a set $[A]^{2}$ is the set of two element subsets of $A$. If $A$ is totally ordered by $<$, we may think of $[A]^{2}$ as $\{(a, b) \in A \times A: a<b\}$. A theorem of

Received by the editors August 9, 1979 and, in revised form, November 14, 1979.

1980 Mathematics Subject Classification. Primary 54A35, 54D18.

Key words and phrases. Club, Martin's Axiom, Dowker space.

'Partially supported by NSF grant MCS 79-01848. 
Dushnik and Miller ${ }^{2}$ asserts that wherever $(A,<)$ has order type $\omega_{1}$ and $f:[A]^{2} \rightarrow\{0,1\}$ then either there is a subset, $S_{0}$, of $A$ of order type $\omega_{1}$ such that $f(a, b)=0$ for all $(a, b) \in\left[S_{0}\right]^{2}$ [we will abbreviate $f((a, b))$ by $\left.f(a, b)\right]$ or there is a subset, $S_{1}$, of order type $\omega+1$ such that $f(a, b)=1$ for all $(a, b) \in\left[S_{1}\right]^{2}$. We abbreviate this theorem by $\omega_{1} \rightarrow\left(\omega_{1}, \omega+1\right)_{2}^{2}$.

Now, assume we are given a space $S(\mathcal{E})$, and a decreasing sequence $\left(H_{n}\right)_{n \in \omega}$ of closed sets with empty intersection. We will use MA $+\neg \mathrm{CH}$ to define a sequence $\left(U_{n}\right)_{n \in \omega}$ of open sets with empty intersection satisfying $U_{n} \supset H_{n}$.

We define $P_{n}$, a poset of approximations to $U_{n}$. Our plan is to define

$$
U_{n}=\bigcup\left\{c_{p}: p \in G\right\}=\bigcup\left\{\bigcup \text { range } b_{p}: p \in G\right\} \text {. }
$$

Let $P_{n}$ be the set of triples $p=\left(a_{p}, c_{p}, b_{p}\right)$, usually abbreviated $(a, c, b)$, satisfying

$$
\begin{aligned}
& a \text { is a finite subset of } S(\mathcal{E})-H_{n} \text {, } \\
& c \text { is a finite subset of } S(\mathcal{L}) \text {, } \\
& b \text { is a function with domain } c, b(x) \in \mathscr{B}_{x} \text {, } \\
& a \cap \bigcup \text { range } b=\varnothing \text {. }
\end{aligned}
$$

We claim that $P$ has property $K$. Let $W$ be an uncountable subset of $P$. First we apply the $\Delta$-system lemma to $\left\{a_{p}: p \in W\right\}$ to get an uncountable $W_{1} \subset W$ such that $\left\{a_{p}: p \in W_{1}\right\}$ is a $\Delta$-system with root $r_{a}$. Similarly obtain an uncountable $W_{2} \subset W_{1}$ such that $\left\{c_{p}: p \in W_{2}\right\}$ is a $\Delta$-system with root $r_{c}$. Next, find an uncountable $W_{3} \subset W_{2}$ such that for all $p, q \in W_{3}, b_{p}\left|r_{c}=b_{q}\right| r_{c}$ and card $c_{p}=k=$ card $c_{q}$. Now we define $W_{4}=\left\{p(\beta): \beta<\omega_{1}\right\}$ by induction on $\beta$ so that the unfixed part of $p(\beta)$ is strictly above the sup of the unfixed parts of $p\left(\beta^{\prime}\right)$, $\beta^{\prime}<\beta$. Precisely, for $x=(n, \alpha) \in S(\mathfrak{L})$ define $h(x)=\alpha$; define $h(a)=$ $\max \{h(x): x \in a\}, h(c)=\max \{h(x): x \in c\}, h(p)=\max \left\{h\left(a_{p}\right), h\left(c_{p}\right)\right\}$. Define $p(\beta)$ so that if $x \in a_{p(\beta)} \cup c_{p(\beta)}$ and $h(x)<\sup \left\{h\left(p\left(\beta^{\prime}\right)\right): \beta^{\prime}<\beta\right\}$, then $x \in r_{a}$ $\cup r_{c}$.

All the above refining has achieved: If $\beta^{\prime}<\beta$, and $p(\beta)$ and $p\left(\beta^{\prime}\right)$ are incompatible, then there is $x \in c_{p(\beta)}-r_{c}$ such that $b_{p(\beta)}(x) \cap a_{p(\beta)} \neq \varnothing$. List each $c_{p(\beta)}$ as $(x(\beta, j))_{j<k}$. We define a function $f_{0}:\left[\omega_{1}\right]^{2} \rightarrow\{0,1\}$ by $f\left(\beta^{\prime}, \beta\right)=0$ iff $b_{p(\beta)}(x(\beta, 0)) \cap a_{p\left(\beta^{\prime}\right)}=\varnothing$. We apply $\omega_{1} \rightarrow\left(\omega_{1}, \omega+1\right)_{2}^{2}$ to get either $S_{0}$ or $S_{1}$.

We claim that we get $S_{0}$ of order type $\omega_{1}$. Aiming for a contradiction, assume $S_{1} \subset W_{4}$ has order type $\omega+1$ and $f\left(\beta^{\prime}, \beta\right)=1$ for all $\left(\beta^{\prime}, \beta\right) \in\left[S_{1}\right]^{2}$. Let $\left(\gamma_{n}\right)_{n<\omega}$ enumerate $S_{1}$ in increasing order. Let $x\left(\gamma_{\omega}, 0\right)=(m+1, \lambda)$. Let $\left(m, \delta_{n}\right) \in b_{p\left(\gamma_{\omega}\right)} \cap$ $a_{p\left(\gamma_{n}\right)}$. Then $\left(\delta_{n}\right)_{n \in \omega}$ is an increasing sequence of elements of $L_{\lambda}$ whose sup is less than $\lambda$. Contradiction.

Define $f_{1}:\left[S_{0}\right]^{2} \rightarrow\{0,1\}$ as we did $f_{0}$ with $x(\beta, 0)$ replaced by $x(\beta, 1)$. The same argument gives that we get a new $S_{0}$ (rather than $S_{1}$ ). We repeat, applying $\omega_{1} \rightarrow\left(\omega_{1}, \omega+1\right)_{2}^{2} k$ times, to get $S^{*} \subset \omega_{1}$ of order type $\omega_{1} ;\left\{p(\beta): \beta \in S^{*}\right\}$ is an

\footnotetext{
${ }^{2}$ Not exactly. Some unidentified person noted that some proofs of the Dushnik-Miller theorem actually give this slightly stronger result. See [W, Theorem 7.4.1]; [F, Theorem 4.5]. It is also a special case of Corollary 1 to Theorem 3.4, [ER].
} 
uncountable subset of $W$ of compatible elements. We have shown that $P_{n}$ has property $K$.

Let $P$ be the product poset $\Pi_{n} P_{n}$. We consider $P$ to be the set of functions $f$ with domain $\omega$ such that $f(n) \in P_{n}$ and $\{n \in \omega: f(n) \neq(\varnothing, \varnothing, \varnothing)\}$ is finite; $f^{\prime}<f$ iff for all $n, f^{\prime}(n)<f(n)$. The following subsets of $P$ are dense: for all $x \in S(\mathfrak{L})$,

$$
D_{x}=\left\{f \in P: \exists n x \in a_{f(n)}\right\}
$$

for all $n \in \omega, x \in H_{n}$

$$
D_{x, n}=\left\{f \in P: x \in c_{f(n)}\right\}
$$

for all $n \in \omega, x \in S(\mathcal{L})$

$$
D_{x, n}^{\prime}=\left\{f \in P: x \in c_{f(n)} \cup a_{f(n)}\right\} .
$$

Since there are $\omega_{1}$ of the above dense subsets, by $\mathrm{MA}+\neg \mathrm{CH}$ there is a filter $G$ on $P$ meeting all of them. For each $n \in \omega$, define $U_{n}=U\left\{c_{f(n)}: f \in G\right\}$. Then $\left(U_{n}\right)_{n \in \omega}$ is a sequence of open subsets with empty intersection satisfying $U_{n} \supset H_{n}$. (The first family of dense sets ensures that $\bigcap_{n \in \omega} U_{n}=\varnothing$; the second that $U_{n} \ni$ $H_{n}$; the third that $U_{n}$ is open.) We have shown that $S(\mathscr{L})$ is countably metacompact.

ADDENDA. 1. A simple modification of the proof shows that, assuming $\mathbf{M A}_{\omega_{1}}$, every subset of $S(\mathcal{E})$ is $G_{\delta}$.

2. P. Nyikos has also asked whether the following statement, $\Sigma$, is a theorem of ZFC.

"There is a ladder system $\mathcal{L}=\left(L_{\lambda}: \lambda \in \omega_{1} \cap\right.$ LIM) such that whenever $x$ is a stationary subset of $\omega_{1}$, then there is $\lambda<\omega_{1}$ such that $x \cap L_{\lambda}$ is infinite."

Negative answers have been given by Komj'ath, Kunen, and the author. Kunen showed that $\mathrm{MA}_{\omega_{1}} \rightarrow \neg \Sigma$, using the product of $\omega$ copies of Wage's poset [Wa]. By a density argument, the union of the countably many "generic" sets is $\omega_{1}$, so at least one must be stationary. The author's negative answer is now a technique looking for an application. The key idea is that the following statement is consistent with ZFC.

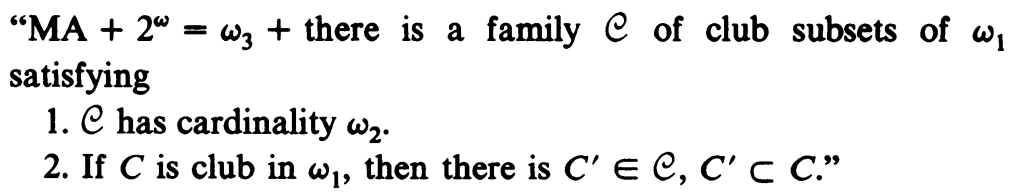




\section{BIBLIOGRAPHY}

[dC] P. de Caux, A collectionwise normal weakly $\theta$-refinable Dowker space which is neither irreducible nor realcompact, Topology Proceedings 1 (1976), 67-78.

[ER] P. Erdös and R. Rado, A partition calculus in set theory, Bull. Amer. Math. Soc. 62 (1956), 427-489.

[F] W. Fleissner, Applications of stationary sets in topology, Surveys in General Topology (G. Reed, Ed.), Academic Press, New York, 1980, pp. 163-183.

[N] P. Nyikos, A topological Litmus paper strip, Notices Amer. Math. Soc. 26 (1979), A-478.

[R] M. E. Rudin, Lectures on set theoretic topology, CBMS Regional Conf. Ser. Math., vol. 23, Amer. Math. Soc., Providence, R. I., 1975.

[Wa] M. L. Wage, Almost disjoint sets and Martin's axiom, J. Symbolic Logic 44 (1979), 313-318.

[W] N. Williams, Combinatorial set theory, North-Holland, Amsterdam, 1977.

Department of Mathiematics, University of Ptttsburgh, Ptttsburgh, Pennsylvania 15261 